\title{
From the gut to bone: connecting the gut microbiota with Th17 T lymphocytes and postmenopausal osteoporosis
}

\author{
Joseph Lorenzo \\ Departments of Medicine and Orthopaedics, UConn Health, Farmington, Connecticut, USA.
}

\begin{abstract}
Osteoporosis is a serious clinical problem that often follows the accelerated bone loss that occurs after the estrogen withdrawal of menopause. In order to better understand the mechanism that produces estrogen withdrawalinduced bone loss, Yu and Pal et al., as reported in this issue of the $J C l$, examined mice that underwent ovariectomy (OVX). In C57BL/6 mice with enhanced Th17 cells in gut tissue, the authors demonstrated that OVX increased migration of TNF-expressing Th17 cells from the gut to the bone marrow. Furthermore, they found that manipulation of the pathways by which lymphocytes migrate and home to bone marrow prevented the increase of $\mathrm{TNF}^{+}$, Th17 cells in bone marrow after OVX in mice and the trabecular, but not cortical, bone loss in this model. These results argue that interactions of the gut microbiota with the immune system are involved in the effects of estrogen withdrawal on trabecular bone.
\end{abstract}

\section{Osteoporosis is initiated by estrogen withdrawal}

Osteoporosis is a disease of low bone mass and/or poor bone quality that can progress to skeletal fractures that occur spontaneously or with minimal impact (1). By age 50 , White women are estimated to have a $15 \%$ to $20 \%$ lifetime risk of a hip fracture and a $50 \%$ risk of an osteoporotic fracture at any site (2). One predisposing factor to the development of osteoporosis in women is menopause. Osteoporosis develops because estrogen withdrawal at menopause initiates a period of accelerated bone loss (1). For many years, estrogen replacement was prescribed as a therapy for postmenopausal osteoporosis. However, estrogen replacement has its own substantial risks (3). Likewise, all currently approved drugs for osteoporosis have substantial, albeit rare, complications asso- ciated with their use, which have limited their acceptance by patients as therapies for osteoporosis (4). Hence, it would seem imperative to understand the mechanisms by which estrogen affects the development of osteoporosis in women in order to identify drug targets and design better therapies for this disease.

It has been known for many years that estrogens regulate the immune system (5) and that immune cells and their products have major effects on bone cells (6). Initial studies in mice and humans demonstrated that proinflammatory cytokines were involved in the development of the bone loss that occurs after estrogen withdrawal (7). However, more recent work has implicated both $\mathrm{T}$ and B lymphocytes ( $\mathrm{T}$ cells and $B$ cells) as mediators of the effects of estrogen on bone mass (8). Bone resorption is facilitated by osteoclasts under the

Related Article: https://doi.org/10.1172/JCl143137

Conflict of interest: The author is a coinventor on two provisional patent applications, "Parmodulin therapy for bone loss" (63001666) and "PRG4 reducing or preventing bone resorption" (63048840).

influence of two cytokines: CSF-1, which is also known as macrophage CSF (M-CSF), and RANKL (9). RANKL, which binds the cognate receptor RANK, is the critical factor that directs the terminal differentiation of multipotential myeloid precursor cells into multinuclear bone-resorbing osteoclasts (10). Both T cells and B cells produce RANKL. However, in a mouse study of ovariectomy-induced (OVX-induced) estrogen withdrawal, it was shown that production of RANKL by B cells rather than $\mathrm{T}$ cells influenced the resultant loss of bone mass (11).

$\mathrm{T}$ cells have a variety of critical functions in the immune system. They are principally involved in initiating adaptive immunity through their ability to respond to antigens presented to them by specialized antigen-presenting cells (12). Multiple types of T cells exist (13). T cells expressing the antigen CD4 have helper activity and are further subdivided into subsets that include Th1, Th2, Tregs, and Th17 cells. Th1 cells secrete INF- $\gamma$ and regulate cell-mediated immunity. Th2 cells secrete IL-4 and IL-10 and influence antibody production. Tregs secrete IL-10 and TGF- $\beta$ and usually inhibit immune responses. In contrast, Th17 cells produce IL-17 and in general are activators of inflammatory responses.

\section{Immune system interactions with gut microbiota affect} bone health

We exist in a sea of microorganisms, the microbiota, that colonize our skin and gastrointestinal tract. A critical role of the immune system is to facilitate the beneficial effects of this symbiotic relationship while simultaneously preventing the invasion of the host by pathogenic organisms in the microbiota (14). It is now clear that the interactions of the immune system with the gut microbiota have profound effects on bone health (15). How- 


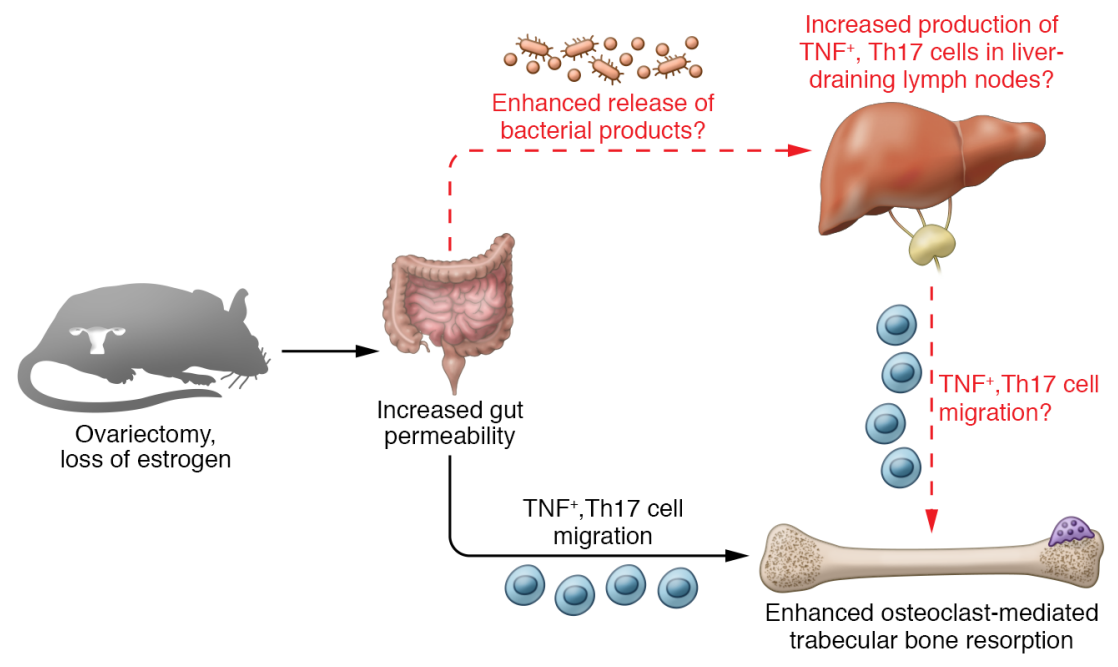

Figure 1. Model for estrogen-withdrawal bone loss. Loss of estrogen after OVX in mice enhances gut permeability, which causes increased release of gut-resident TNF+, Th17 cells. These T cells migrate to bone and enhance osteoclast-mediated trabecular bone resorption. It is also possible that enhanced release of gut bacterial products after OVX causes increased production of TNF+, Th17 cells in peripheral lymphoid organs such as liver-draining lymph nodes. $\mathrm{TNF}^{+}$, Th17 cells produced in response at these sites may also migrate to bone and affect trabecular bone resorption.

ever, comparison of the bone phenotype of mice raised in germ-free conditions with mice raised in conventional housing or with germ-free mice that were recolonized with a conventional microbiota has yielded conflicting results. These differences appear to depend on the strain of mice, the length of time after colonization that mice were examined, and the composition of the microbiota in the mice (15). Li et al. found that in a model of estrogen deficiency, germ-free mice failed to lose trabecular bone mass (16). Li et al. also demonstrated that treatment of conventionally housed mice with certain strains of probiotic bacteria prevented trabecular bone loss after estrogen withdrawal (16).

In this issue of the JCI, Yu and Pal et al. (17) follow up the Li et al. paper (16), exploring the role that Th17 cells in the gut have in mediating bone loss related to estrogen withdrawal in mice. The researchers used C57BL/6 mice from the Jackson Laboratory (JAX), which have a relatively low amount of Th17 cells in their gut tissue (18). In order to boost Th17 cell levels in the gastrointestinal tract, the mice were gavaged with a suspension of stool from mice previously monocolonized with segmented filamentous bacteria (SFB). This process markedly increases the number of Th17 cells in gut tissue (18).

Using C57BL/6 Kaede mice, which allow photoconversion of a fluorescent marker gene in all cells from green to red (19), Yu and Pal et al. convincingly demonstrated that OVX facilitated the migration of Th17 cells and $\mathrm{TNF}^{+} \mathrm{T}$ cells from the gut to the bone marrow. They also adaptively transferred splenic Th17 cells, which express a GFP marker under the direction of the Il17a gene, into WT and TNFdeficient $\left(\mathrm{TNF}^{-/}\right)$mice that had undergone sham operation or OVX two weeks earlier. These studies showed that deletion of TNF abrogated the ability of Th17 cells to home to bone marrow after OVX (17).

The authors went on to demonstrate that expression of the chemokine ligand CCL20, which is known to guide the homing of Th17 cells to bone marrow, is increased after OVX in WT but not $\mathrm{TNF}^{-1}$ mice. Additional studies found that inhibition of S1P receptor 1 (S1PR1) with FTY720 blocked the ability of $\mathrm{TNF}^{+}$and $\mathrm{Th} 17$ cells to accumulate in bone marrow after OVX and the ability of OVX to decrease trabecular but not cortical bone mass in mice. Yu and Pal et al. also used a neutralizing antibody to CCL2O to block the ability of cells expressing both TNF and IL-17 to accumument, anti-CCL2O treatment prevented the OVX-induced loss of trabecular but not cortical bone mass in mice. Finally, the authors examined the effects of OVX on mice with CXCR3 deficiency (CXCR3 ${ }^{-/}$) because CXCR3 is a receptor on $\mathrm{T}$ cells that late in bone marrow. As with FTY720 treat- guides their entry into distant lymphoid organs. They found that $\mathrm{CXCR}^{-/-}$mice did not alter bone marrow $\mathrm{TNF}^{+} \mathrm{T}$ cells or $\mathrm{Th} 17$ cells with OVX. In addition, OVX did not decrease trabecular bone mass in CXCR3 ${ }^{-/-}$ mice. However, as with inhibition of S1PR1 and CCL2O, OVX did induce cortical bone loss in $\mathrm{CXCR3}^{-/-}$mice, which was similar to that of WT mice (17).

\section{Conclusions and remaining questions}

Yu and Pal et al. (17) speculate that migration of $\mathrm{TNF}^{+}$, Th17 cells from the gut to the bone marrow mediated at least the trabecular bone loss of estrogen withdrawal. The work of Yu and Pal et al. (17) and of Li et al. (16) argues strongly that the composition of the microbiota and trafficking of lymphocytes is involved in some effects of OVX on bone mass. However, there remain a number of questions. The first revolves around the use of SFB to enhance levels of Th17 cell in the gut tissue of all of the mice in the Yu and Pal et al. study (17). If migration of gut $\mathrm{TNF}^{+}$, Th17 cells to the bone marrow mediates the effects of OVX on trabecular bone mass as $\mathrm{Yu}$ and $\mathrm{Pal}$ et al. (17) hypothesize, then why do SFB- JAX C57BL/6 mice lose trabecular bone mass at a rate similar to that of $\mathrm{SFB}^{+} \mathrm{JAX} \mathrm{C} 57 \mathrm{BL} / 6$ mice (20)? As mentioned above, $\mathrm{SFB}^{-} \mathrm{JAX}$ C57BL/6 mice have markedly fewer Th17 cells in their gut tissue than $\mathrm{SFB}^{+} \mathrm{JAX}$ C57BL/6 mice. Hence, one might anticipate a proportional decrease in the ability of $\mathrm{SFB}^{-} \mathrm{JAX}$ C57BL/6 mice to lose bone mass with OVX if the hypothesis of Yu and $\mathrm{Pal}$ et al. is correct. It would be interesting to compare the effects of OVX on bone marrow $\mathrm{TNF}^{+}$, Th17 cells in JAX C57BL/6 mice that are $\mathrm{SFB}^{-}$and $\mathrm{SFB}^{+}$. Studies that perform a dose response of SFB addition to the gut microbiota of JAX C57BL/6 mice and then correlate levels of gut and bone marrow Th17 cells with the effects of OVX on trabecular bone loss would be of value. It is also unclear why modulating lymphocyte migration only affected trabecular bone loss after OVX in the Yu and Pal et al. study (17) or why other authors have found contradictory results $(21,22)$. Could these inconsistencies result from differences in the microbiotas of experimental animals?

Notably, SFB itself affects bone mass. The presence of SFB within the gut microbiota of C57BL/6 mice (from Taconic) sub- 
stantially decreased trabecular bone mass and increased liver production of certain acute-phase reactants. Further, the liverdraining lymph nodes and bone marrow both showed increases in the percentages of Th17 cells (23). Osteoclast number was also increased in the bones of $\mathrm{SFB}^{+}$mice. Extending these results to the work of $\mathrm{Yu}$ and $\mathrm{Pal}$ et al. may offer an additional mechanism for estrogen-withdrawal bone loss (Figure 1). Since it is known that OVX enhances gut permeability of bacterial products (16), it's possible that enhanced release of gut bacterial products alter immune cells in the liver and other lymphoid organs to influence the effects of estrogen withdrawal on bone mass.

Ultimately, these concepts need to be examined in human studies. While modulation of immune cell function is possible, this therapy may have untoward effects that will limit its use for osteoporosis. In contrast, manipulation of the microbiota with either pre- or probiotics may have utility both to reduce generalized inflammation and to treat osteoporosis with minimal side effects (24).

\section{Acknowledgments}

This work was supported by grants from the National Institute of Arthritis, Musculoskeletal and Skin Diseases of the United States NIH (R56AR074391 and R21AR076671).

Address correspondence to: Joseph Lorenzo, MSI 4th floor, N4054, MC: 4037,
UConn Health, 263 Farmington Avenue, Farmington, Connecticut 060304037, USA. Phone: 860.679.8199; Email: jlorenzo@uchc.edu.

1. Black DM, Rosen CJ. Clinical practice. Postmenopausal osteoporosis. N Engl J Med. 2016;374(3):254-62.

2. Cummings SR, Melton LJ. Epidemiology and outcomes of osteoporotic fractures. Lancet. 2002;359(9319):1761-1767.

3. Rossouw JE, et al. Risks and benefits of estrogen plus progestin in healthy postmenopausal women: principal results From the Women's Health Initiative randomized controlled trial. JAMA. 2002;288(3):321-333.

4. Khosla S, Shane E. A crisis in the treatment of osteoporosis. J Bone Miner Res. 2016;31(8):1485-1487.

5. Gameiro C, Romao F. Changes in the immune system during menopause and aging. Front Biosci (Elite Ed). 2010;2:1299-1303.

6. Lorenzo J, et al. Osteoimmunology: interactions of the bone and immune system. Endocr Rev. 2008;29(4):403-440.

7. Clowes JA, et al. The role of the immune system in the pathophysiology of osteoporosis. Immunol Rev. 2005;208:207-227.

8. Almeida M, et al. Estrogens and androgens in skeletal physiology and pathophysiology. Physiol Rev. 2017;97(1):135-187.

9. Teitelbaum SL. Bone resorption by osteoclasts. Science. 2000;289(5484):1504-1508.

10. Boyle WJ, et al. Osteoclast differentiation and activation. Nature. 2003;423(6937):337-342.

11. Onal M, et al. Receptor activator of nuclear factor $\kappa \mathrm{B}$ ligand (RANKL) protein expression by B lymphocytes contributes to ovariectomy-induced bone loss. J Biol Chem. 2012;287(35):29851-29860.

12. Pacifici R. Role of T cells in ovariectomy induced bone loss-revisited. J Bone Miner Res.
2012;27(2):231-239.

13. Kumar BV, et al. Human T cell development, localization, and function throughout life. Immunity. 2018;48(2):202-213.

14. Zhou Y, et al. Biogeography of the ecosystems of the healthy human body. Genome Biol. 2013;14(1):R1.

15. Yan J, Charles JF. Gut microbiome and bone: to build, destroy, or both? Curr Osteoporos Rep. 2017;15(4):376-384.

16. Li JY, et al. Sex steroid deficiency-associated bone loss is microbiota dependent and prevented by probiotics. J Clin Invest. 2016;126(6):2049-2063.

17. Yu M, et al. Ovariectomy induces bone loss via microbial-dependent trafficking of intestinal $\mathrm{TNF}^{+} \mathrm{T}$ cells and Th17 cells. JClin Invest. 2021;131(4): e143137.

18. Ivanov II, et al. Induction of intestinal Th17 cells by segmented filamentous bacteria. Cell. 2009;139(3):485-498.

19. Tomura M, et al. Activated regulatory $\mathrm{T}$ cells are the major T cell type emigrating from the skin during a cutaneous immune response in mice. J Clin Invest. 2010;120(3):883-893.

20. Gao Y, et al. Estrogen prevents bone loss through transforming growth factor beta signaling in T cells. Proc Natl Acad Sci U S A. 2004;101(47):16618-16623.

21. Lee SK, et al. T lymphocyte-deficient mice lose trabecular bone mass with ovariectomy. J Bone Miner Res. 2006;21(11):1704-1712.

22. Scheffler JM, et al. Interleukin 17A: a Janusfaced regulator of osteoporosis. Sci Rep. 2020;10(1):5692.

23. Hathaway-Schrader JD, et al. Specific commensal bacterium critically regulates gut microbiota osteoimmunomodulatory actions during normal postpubertal skeletal growth and maturation. JBMR Plus. 2020;4(3):e10338.

24. Rizzoli R, Biver E. Are probiotics the new calcium and vitamin D for bone health? Curr Osteoporos Rep. 2020;18(3):273-284 